\title{
Two new species and one new combination of Helina Robineau-Desvoidy, 1830 (Diptera: Muscidae) from China
}

\author{
Ming-Fu Wang*, Chen Sun \& Rong-Rong Wang
}

Wang, M. F., Sun, C. \& Wang R. R. 2012: Two new species and one new combination of Helina Robineau-Desvoidy, 1830 (Diptera: Muscidae) from China. Entomol. Fenn. 23: 107-112.

Two new species of the genus Helina Robineau-Desvoidy, 1830 from Sichuan, China are described and illustrated, i.e. Helina fulvibasicosta Ming-Fu Wang sp. n. and Helina flavipes Ming-Fu Wang \& Chen Sun sp. n. After re-examining the holotype, Helina occidentalisinica Feng, Shi \& Li, 2005 is transferred to the genus Hebecnema Schnabl.

M. F. Wang \& C. Sun, College of Chemistry and Life Sciences, Shenyang Normal University, Shenyang 110034, China; *Corresponding author's e-mail: wangmingfu403@163.com

R. R. Wang, Key Laboratory of Zoological Systematics and Evolution, Institute of Zoology, Chinese Academy of Sciences, Beijing 100101, China

Received 30 December 2011, accepted 24 April 2012

\section{Introduction}

The genus Helina Robineau-Desvoidy, 1830 is the second largest genus of Muscidae, in which over 570 species are currently known worldwide. Due to the facts that many specialists consider that Helina is a "rest-all" repository and Helina includes so many species, it is difficult to identify the species correctly.

Since the beginning of the 20th century, 231 species of Helina have been identified in China by 47 Chinese colleagues in 30 different organizations, accounting for $40 \%$ of the species worldwide, among which 199 are endemic to China. Since 2000, we have been engaged in faunal studies of this group in China, especially focusing on the revision work of the genus. While sorting and identifying Helina from material in the College of Chemistry and Life Sciences, Shenyang Normal University, Shenyang, China, we found two new species collected from Sichuan. In this paper, we describe those two new species, and provide a new combination for Helina occidentalisinica Feng, Shi \& Li, 2005.

\section{Material and methods}

Morphological terminology of McAlpine (1981) is followed. Absolute measurements are used for body length in millimeters $(\mathrm{mm})$.

Abbreviations used for characters include: $a=$ anterior seta, $a c r=$ acrostichal seta, $a d=$ anterodorsal seta, $a v=$ anteroventral seta, $d=$ dorsal seta, $d c=$ dorsocentral seta, $i a=$ intra-alar seta, $p=$ posterior seta, $p d=$ posterodorsal seta, $p r a=$ prealar seta, $p v=$ posteroventral seta, and $v=$ ventral seta.

All specimens studied are deposited in the College of Chemistry and Life Sciences, Shenyang Normal University, Shenyang and in the Shanghai Entomological Museum, Chinese Academy of Sciences, Shanghai (SEMCAS). The specimens from SEMCAS are noted. 

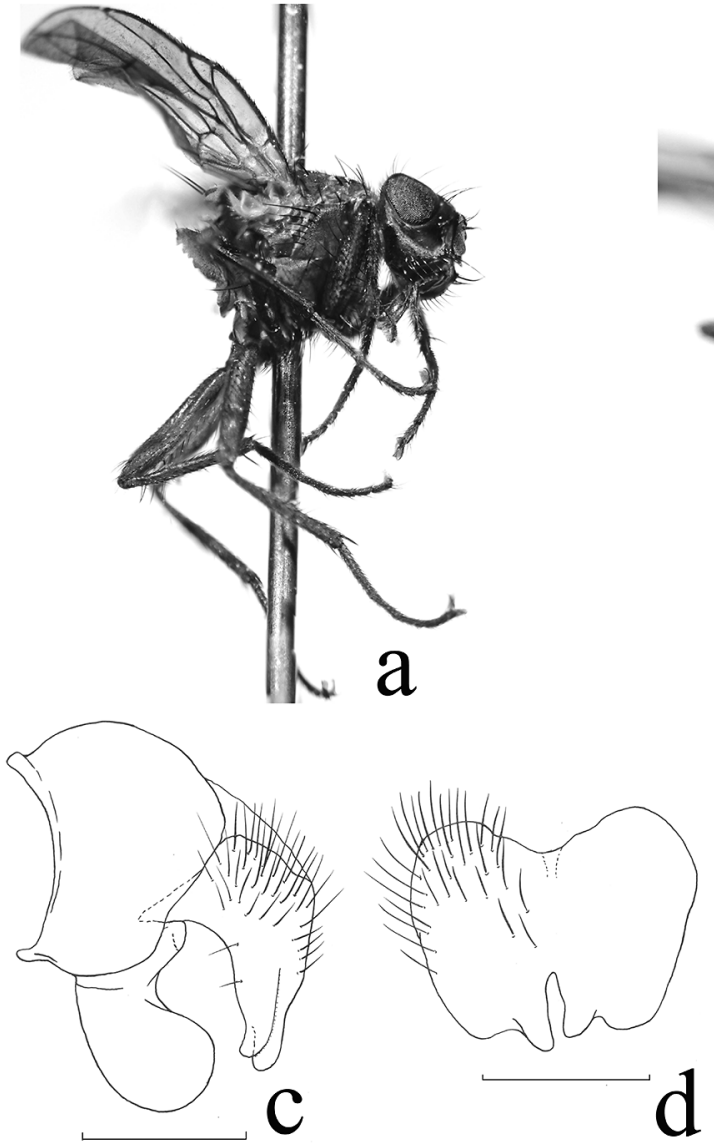
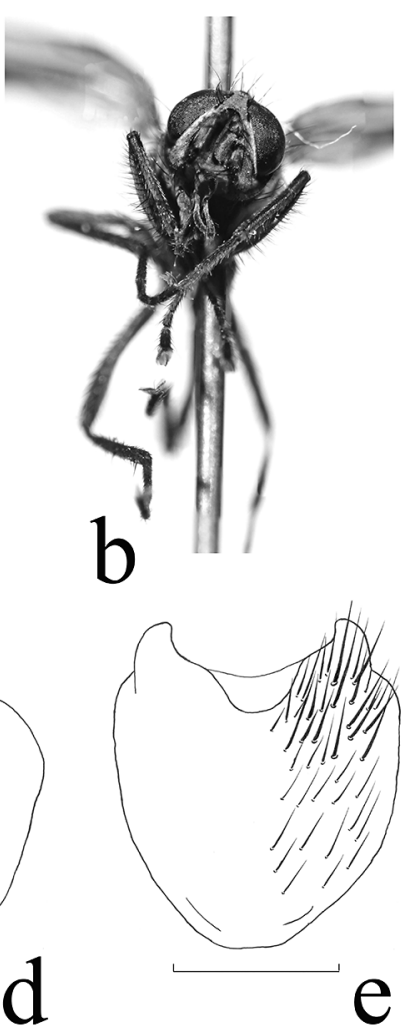

Fig. 1. Helina fulvibasicosta Ming-Fu Wang sp. n., male holotype. -a. Body, lateral view. - b. Body, anterior view. - c. Terminalia, lateral view, scale $0.25 \mathrm{~mm}$. -d. Cercal plate, ventral view, scale 0.25 mm. - e. Sternite 5, scale $0.4 \mathrm{~mm}$.

\section{Taxonomy}

\subsection{Helina fulvibasicosta Ming-Fu Wang sp. n. Fig. 1}

Type material. Holotype, male, China: Mt. Balang $\left(30^{\circ} 54^{\prime} \mathrm{N}, 102^{\circ} 54^{\prime} \mathrm{E}\right)$, alt. 4,600 $\mathrm{m}$ a.s.l., 8.VIII.2005, leg. M. F. Wang. Paratype, 1 male, same data as holotype.

Description. Male. Body length $6.2 \mathrm{~mm}$.

Head. Eyes bare; facets not enlarged; frons moderately wide, about 1.2 times of distance between posterior ocelli, and 1.6-1.8 times as wide as antennal first flagellomere; frontal vitta black, at the narrowest point about 4 or 5 times of frontoorbital; frontal setae 7 , situated on lower $2 / 3$, reaching the level of anterior the ocellus, orbital setae absent; ocellar setae longer than the lower frontal seta; fronto-orbital plate and parafacial with greyish-brown pruinosity, parafacial about 1.2-1.3 times as wide as flagellomere; antenna black, flagellomere 2.5 times as long as broad, arista short plumose, the longest hair about $2 / 3$ of flagellomere-width; lunule dark brown, epistoma not projecting, vibrissal angle behind profrons in profile; gena with grey pruinosity, the upper margin of gena dark reddish-brown, gena about $1 / 3$ of eye-height, the upper margin of gena with a row of upcurved setae, gena and metacephalon with black hairs; proboscis short, labella large, prementum with greyish thin pruinosity, 2 times longer than width; palpus black and claviform, slightly oblate at distal part and a little longer than prementum.

Thorax. Ground colour black with thinner grayish-white pruinosity, scutum with four obscure longitudinal vittae, the inside vittae not reaching to the scutellum suture; presutural $a c r 3$ rows, posterior acr 1, dc 2+3, ia $0+2$; pra strong, distinctly longer than posterior notopleural seta; notopleuron, lateral margin and lower surface of scutellum bare; basisternum, proepisternum, 
anepimeron, meron and katepimeron bare; katepisternal bristle 2:2, spiracles brown, calypters small.

Wing. Brown, veins brown, wing-base yellow, basicosta yellowish-brown, costal spine inconspicuous; vein Sc not cured bow-like, node of Rs bare on ventral and dorsal surfaces; vein $M$ straight, vein $\mathrm{R} 4+5$ and $\mathrm{M}$ well separated from each other on apixal part; cross-vein r-m robust, veins $m-m$ straight, cross veins without conspicuous cloud; calypters yellow, the lower one projecting; halteres brownish-yellow.

Legs. Black, fore tibia with one median $p$, and several long hairs on ventral surface; mid femur without conspicuous $a v$ row but with a complete and long $p v$ row, $a d$ row on basal $1 / 2$; mid tibia with $1 a d, 2$ or $3 p d$; hind coxa bare on posterior surface; hind femur with complete and long $a v$ row, with 6 or $7 p v$ setae on 1/5 of distal on poster oventral surface; hind tibia with $3 a d$, without $p d$, hind tibia with 2 rows of slender and longer setae on $a v$ and $p v$ surfaces, the length of setae equal to the width of hind femur.

Abdomen. Oval, black in ground colour, with dark grayish-brown pruinosity; tergites 2 to tergite 4 each with 1 pair of big and subquadrate dark spots, slightly extended outside on latter margin; tergite 5 without spots; each side of sternite 1 with one seta, sternite 5 and terminalia of male as shown in Fig. 1.

Female. Unknown.

Etymology. The species name is from Latin and refers to the yellow colour of basicosta.

Remarks. This is a rare species. It has yellow legs and a yellow basicosta, so it can be easily separated from most Palaearctic Helina species, except $H$. altica Wang, Xue \& Wang, 2005, which has been described from Mt. Doxong La, Xizang, China. H. fulvibasicosta can be incorporated into the male key of Xue et al. (2005) as follows:

73. Hind tibia with dense but untidy rows of long hairs on anteroventral and posteroventral surfaces simultaneously

$74 \mathrm{a}$

74a. Tibia yellow; crossvein $\mathrm{r}-\mathrm{m}$ and $\mathrm{dm}-\mathrm{cu}$ clouded; frons narrow, about as wide as anterior ocellus; prealar seta about $1 / 2$ length of the second notopleural bristle

H.cothurnata (Rondani)
- Leg wholly black; crossvein r-m and dm-cu unclouded; frons broader, wider than the distance between outer margins of posterior ocelli; parafacial as wide as antennal flagellomere; prealar seta as long as the second notopleural bristle

74b. Parafacial as wide as antennal flagellomere; prealar seta as long as the second notopleural bristle; frontal vitta as wide as or narrower than anterior ocellus; outside proceses longer than inner proceses in the distal of cersus

H. celsa (Harris)

- Parafacial wider than antennal flagellomere; prealar seta longer than the second notopleural bristle; frontal vitta more than twice as wide as anterior ocellus; the length of outside proceses equal to or shorter than inner proceses in the distal of cersus $74 \mathrm{c}$

$74 \mathrm{c}$. The longest hair of arista as long as one-third width of antennal flagellomere; basicosta dark brown, crossveins r-m clouded, fore tibia without median $p$, mid tibia without $a d$, sternite 1 bare H. altica Wang, Xue \& Wang The longest hair of arista as long as two-third width of antennal flagellomere; basicosta yellowish brown, crossveins $\mathrm{r}-\mathrm{m}$ without cloud; fore tibia with 1 strong median $p$, mid tibia with 1 long $a d$, sternite 1 with hairs

H. fulvibasicosta $\mathbf{s p .} \mathbf{n}$.

\subsection{Helina flavipes Ming-Fu Wang \& Chen Sun, sp. n. Fig. 2}

Type material. Holotype, male, China: Mt. Erlang (306'N, $\left.102^{\circ} 32^{\prime} 24^{\prime} ' \mathrm{E}\right)$, alt. 1,900-2,930 m a.s.1., 3.VII.2006, leg. L.Y. Feng. Paratypes, 1 male, same data as holotype.

Description. Male. Body length $6.0 \mathrm{~mm}$.

Head. Eyes with obviously light brownish hairs, facets enlarged on anterior margin in upper part; frontal narrow, frons-width at the narrowest part nearly equal to the distance between anterior ocellus, frontal setae 5-6, situated on the lower half of frons, orbital seta absent, ocellus seta shorter than lower frons seta; fronto-orbital plate and parafacial with greyish-brown pruinosity, parafacial slightly narrower than flagellomere, antenna black, flagellomere 3 times as long as broad, arista long plumose, the longest hair 

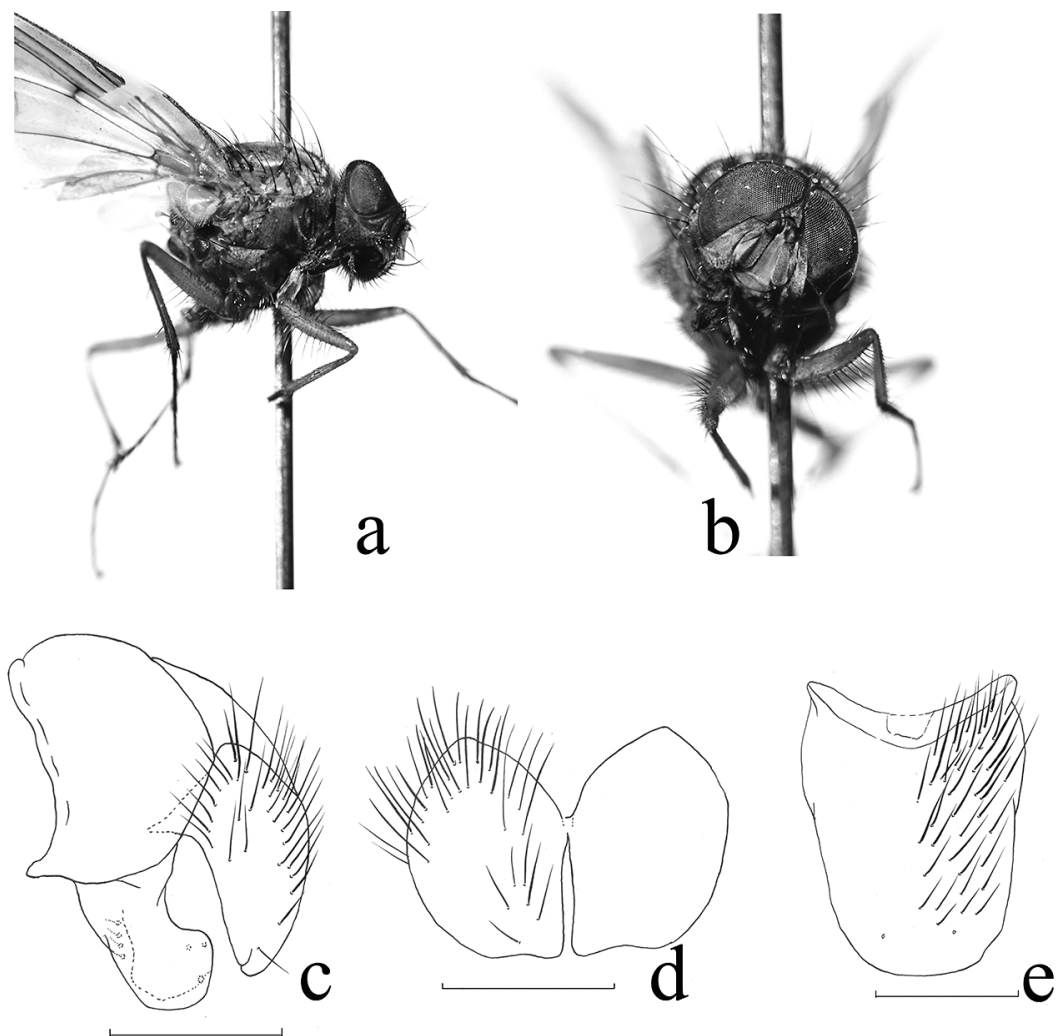

Fig. 2. Helina flavipes Ming-Fu Wang \& Chen Sun, sp. n., male holotype. - a. Body, lateral view. - b. Body, anterior view. - c. Terminalia, lateral view, scale 0.25 $\mathrm{mm}$. - d. Cercal plate, ventral view, scale 0.25 $\mathrm{mm}$. - e. Sternite 5, scale $0.4 \mathrm{~mm}$.

slightly shorter than flagellomere; lunule yellow, arista short plumose; epistoma not projecting, vibrissal angle behind profrons in profile; upper margin of genae with a row of upcurved setae; gena and postgena with black hairs; gena with grayish-brown pruinosity; proboscis short, with grayish-brown pruinosity, prementum 2 times longer than wide; labella large, palpus black, except on basal part brown, length longer than prementum.

Thorax. Ground-colour black with dark greyish-brown pruinosity, scutum with four dark longitudinal vittae, the inside vittae nearly reaching to the middle of scutellar suture; 5-6 rows of presutural $a c r$, posterior $a c r 1, d c 2+4$, ia $0+2$, pra strong, slightly shorter than posterior notopleural seta; notopleuron, lateral margin and lower surface of scutellum bare; basisternum, proepisternum, anepimeron, meron and katepimeron bare; katepisternal bristle $2: 2$, spiracles dark brown and large.

Wing. Light brown, veins dark brown, wingbase and other position with same colour, tegula and basicosta dark brown, costal spine strong, shorter than the length of cross-veins r-m, vein Sc cured bow-like; node of Rs bare on ventral and dorsal surfaces; vein $\mathrm{m} 1+2$ stright, vein $\mathrm{m} 1+2$ and vein $\mathrm{R} 4+5$ well separated from each other at apex, cross-vein r-m robust; crossveins with obvious cloud, crossveins $\mathrm{m}-\mathrm{m}$ projecting forward in middle, with little cloud; calypters yellow, the lower one projecting tongue-like; halteres yellowish-orange.

Legs. Femora and tibiae yellow, tarsi black; fore tibia with one median $p$, mid femur with complete $a v$ row, fine and obvious; $a d$ row present on basal $3 / 5$, median $p v$ row long, ones on distal part slightly shorter, mid tibia with 3 long $p$, hind coxa bare on posterior surface, hind femur with long $a v$ and $a d$, obvious and long hair-like $p v$ row on distal $1 / 3$, the longest seta equal to or shorter than the width of hind femur, hind tibia with $3 a v, 2 a d$, with 6 or 7 short semierect setae row behind median part.

Abdomen. Oval, black in ground colour, with dark golden brown pruinosity; tergites 3 and 4 each with a pair of big and subrectangular dark patches without pruinosity, sternite 1 bare. 
Fig. 3. Hebecnema occidentalisinica (Feng, Shi \& Li, 2005), male holotype. - a. Terminalia, lateral view. - b. Cercal plate, ventral view. - c. Sternite 5. (Adapted from Feng et al. 2005).

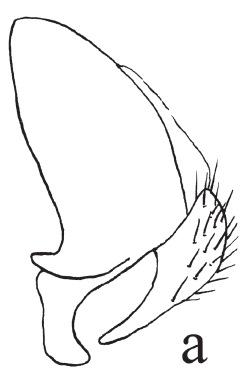

Female. Unknown.

Etymology. The species name is derived from Latin, referring to its yellow legs.

Remarks. H. flavipes sp. n. differs from other Helina species in its light body colour, with ten rows of acr setae, and lack of $d c$ setae. It can be incorporated into the male key of Xue et al. (2005) as follows:

104a. Fore tibia with 1 median $p$

- $\quad$ Fore tibia without median $p$

104b. Frons about 1.5 times as wide as antennal flagellomere; parafacial nearly $1.75-2.0$ times as wide as antennal flagellomere; costal spine inconspicuous; hind tibia with 7-8 av H. bibreviplumosa Xue \& Feng

105. Frons narrower than the width of anterior ocellus; parafacial narrower than the width of antennal flagellomere; costal spine con-
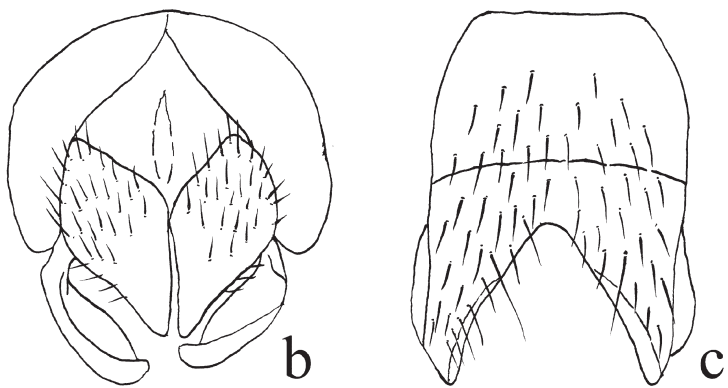

spicuous, shorter than the length of crossvein $\mathrm{r}-\mathrm{m}$; hind tibia with 3 av

H. flavipes sp. n.

\subsection{Hebecnema occidentalisinica} (Feng, Shi \& Li, 2005), comb. n. Fig. 3

Helina occidentalisinica Feng, Shi \& Li, 2005: 93, fig. 1-4.

Type material. Holotype, male, China: Mt. Erlang, alt. 2,760 m a.s.1., 21. VI. 1982, leg. P. Shi (SEMCAS).

Remarks. Emden (1951) divided Helina into five subgenera, i.e. Helina s. str., Euspilaria Malloch, Helinella Malloch, Hebecnema Schnabl and Mydhelina Emden. However, some of the groups treated as subgenera by Emden are now recognised as genera, i.e., Hebecnema Schnabl, while other taxa described as genera by Emden

Table 1. Diagnostic characters between Hebecnema Schnabl and Helina Robineau-Desvoidy.

\begin{tabular}{|c|c|c|}
\hline & Hebecnema & Helina \\
\hline $\begin{array}{l}\text { Length of anterodorsal } \\
\text { seta in hind tibia on apex part }\end{array}$ & At most equal to width of tibia & Longer than width of tibia \\
\hline Upper proepimeral seta & Absent or hair-like & Usually distinct or strong \\
\hline Prealar seta & Absent or hair-like & $\begin{array}{l}\text { Usually obviously present or strong, } \\
\text { few hair-like }\end{array}$ \\
\hline Abdomonial tergites & $\begin{array}{l}\text { Without pairs of spots, } \\
\text { at most with a median vitta }\end{array}$ & $\begin{array}{l}\text { Usually with pairs of spots, } \\
\text { few species with median vitta }\end{array}$ \\
\hline Katepisternal seta & Usually 1:2 & Usually $2: 2$ \\
\hline Width of parafacial & $\begin{array}{l}\text { Narrower than width } \\
\text { of antennal flagellomere }\end{array}$ & $\begin{array}{l}\text { Equal to or wider than width } \\
\text { of antennal flagellomere }\end{array}$ \\
\hline Head in profile view & $\begin{array}{l}\text { Upper part of head with a } \\
\text { slightly flattened appearance }\end{array}$ & $\begin{array}{l}\text { Upper part of head with a raised } \\
\text { appearance }\end{array}$ \\
\hline Sternite 5 of abdomen & Lateral lobe usually raised & Lateral lobe often flattened \\
\hline Surstylus in profile view & Slender and long & Thicker and shorter \\
\hline
\end{tabular}


have been synonymized with Helina (see Pont 1977, 1980, 1986). Hennig (1957-1958), Fan $(1965,1992)$ and Pont $(1972,1980,1986)$ have all accepted Hebecnema Schnabl as an independent genus. The genus Hebecnema can be differentiated from Helina as presented in Table 1.

Based on re-examination of the holotype of $H$. occidentalisinica and the original description of Feng et al. (2005), we found the main characters of this species different from Helina. The diagnostic characters, including smaller body, upper part of head with a slightly flattened appearance in profile view, parafacial narrower, without $p r a$, katepisternal seta 1:2, mid tibia with $2 p$, hind tibia with $1 a v$ and $1 a d$, are the main diagnostic characters of Hebecnema. According to the morphology of terminalia, we also found that the main characters of $H$. occidentalisinica, including the distal part of terminalia slightly protecting, cerci slender and constricted in profile view, tergite 5 are all in accordance with Hebecnema. We conclude that $H$. occidentalisinica should be assigned to Hebecnema.

Acknowledgments. This study was supported by the National Nature Science Foundation of China (No. 31071957, 30900145, 30770252). We are very grateful to Dr. A. C. Pont (Oxford University Museum of Natural History, Oxford, UK) for his invaluable help over many years. We also owe our gratitude to Prof. Wan-Qi Xue, Mr Li-Yong Feng, Ms Jing Wang and Ms Shuai Wang (College of Chemistry and Life Sciences, Shenyang Normal University), and Mr Qi-ke Wang (Beijing Forestry University), who gave us invaluable help with this study.

\section{References}

Emden, F. I. 1951: Muscidae C. - Scatophaginae, Anthomyiinae, Lispinae, Fanniinae and Phaoniinae. - Ru- wenzori Expedition 1934-1935 2: 325-710, 710 pp.

Fan, Z. D. 1965: Key to the common flies of China, first edition. - Science Press, Beijing, 330 pp. [In Chinese.]

Fan, Z. D. 1992: Key to the common flies of China, second edition. - Science Press, Beijing, 992 pp. [In Chinese. ]

Feng, Y., Shi, P. \& Li, G. 2005: Two new species of Genus Helina from western Sichuan, China (Diptera: Muscidae). - Chinese Journal of Vector Biology and Control 16: 93-94.

Hennig, W. 1957-1958: Muscidae. — In: Lindner, E. (ed.), Die Fliegen der palaearktischen Region. 63b: 145232. Schweizerbart, Stuttgart. 1955-1964, 1100 pp.

McAlpine, J. F. 1981: Morphology and terminology adults. - In: McAlpine, J. F., Peterson, B. V., Shewell, G. E., Teskey, H. J., Vockeroth, J. R. \& Wood, D. M. (eds.), Manual of Nearctic Diptera, 1. Research Branch, Agriculture Canada Monograph 27: 9-63. Canadian Government Publishing Center, Hull, 684 pp.

Pont, A. C. 1972: Family Muscidae. - In: Papavero, N. (ed.), A Catalogue of the Diptera of the Americas South of the United States, 97: 1-111. Museu de Zoologia, Universidade de São Paulo, São Paulo, 111 pp.

Pont, A. C. 1977: Family Muscidae. - In: Delfinado, M. D. \& Hardy, D. E. (eds.), A catalogue of the Diptera of the Oriental Region. Volume 3, Suborder Cyclorrhapha (excluding Division Aschiza): 451-523. University Press of Hawaii, Honolulu, 762 pp.

Pont, A. C. 1980: Family Muscidae. - In: Crosskey, R. W. (ed.), Catalogue of the Diptera of the Afrotropical Region: 721-761. British Museum (Natural History), London, $1437 \mathrm{pp}$.

Pont, A. C. 1986: Family Muscidae. — In: Soós, Á. \& Papp, L. (eds.), Catalogue of Palaearctic Diptera. Volume11, Scathophagidae-Hypodermatidae: 57-215. Akadémiai Kiadó, Budapest, 346 pp.

Wang, M. F., Xue, W. Q. \& Wang, R. R. 2005: A new species of Helina R-D. from Qinghai-Xizang Plateau, China (Diptera: Muscidea). — Acta Zootaxonomica Sinica 30: 191-193.

Xue, W. Q., Feng, Y., Tong, Y. F. 2005: A taxonomic study on Helina R.-D. (Diptera: Muscidae) from China. Oriental Insects 39: 35-62. 